\title{
Identification of a novel homozygous variant in the CNGA1 gene in a Chinese family with autosomal recessive retinitis pigmentosa
}

\author{
LE WANG $^{1 *}$, TONGDAN ZOU ${ }^{2 *}$, YONGQIONG LIN ${ }^{2}, \mathrm{LING} \mathrm{LI}^{2}$, PENG ZHANG $^{3}$, \\ $\mathrm{BO} \mathrm{GONG}^{2,4}$, JILONG HAO ${ }^{1}$ and HOUBIN ZHANG ${ }^{2,4}$
}

\author{
${ }^{1}$ Department of Ophthalmology, The First Hospital of Jilin University, Changchun, Jilin 130103; ${ }^{2}$ Key Laboratory for \\ Human Disease Gene Study of Sichuan Province and Department of Laboratory Medicine, Sichuan Academy of Medical \\ Sciences and Sichuan Provincial People's Hospital, School of Medicine, University of Electronic Science and \\ Technology of China, Chengdu, Sichuan $610072 ;{ }^{3}$ Diagnosis Center, The First Hospital of Jilin University, \\ Changchun, Jilin 130103; ${ }^{4}$ Institute of Laboratory Medicine, Sichuan Provincial \\ People's Hospital, Chengdu, Sichuan 610072, P.R. China
}

Received January 12, 2020; Accepted June 12, 2020

DOI: $10.3892 / \mathrm{mmr} .2020 .11331$

\begin{abstract}
Retinitis pigmentosa (RP) is a complex group of hereditary retinal dystrophies. Although $>60$ genes have been identified to be associated with non-syndromic RP, the exact genetic variant remains elusive in numerous cases of RP. In the present study, a Chinese pedigree affected by RP with autosomal recessive inheritance, including a total of seven members with one affected patient and six unaffected individuals, was recruited. Comprehensive ophthalmic examinations were performed on the proband and the proband's unaffected daughter. Genomic DNA was extracted from peripheral blood. Whole-exome sequencing (WES) was performed for the affected individual. The candidate pathogenic variant was verified by direct Sanger sequencing. The affected individual presented with classical clinical symptoms of RP. A novel homozygous variant, c.265delC (p.L89Ffs*3) in the cyclic nucleotide-gated channel subunit $\alpha$ 1 gene was identified in the affected patient. This homozygous
\end{abstract}

Correspondence to: Dr Jilong Hao, Department of Ophthalmology, The First Hospital of Jilin University, 71 Xinmin Da Jie, Changchun, Jilin 130103, P.R. China

E-mail: 52723060@qq.com

Dr Houbin Zhang, Key Laboratory for Human Disease Gene Study of Sichuan Province and Department of Laboratory Medicine, Sichuan Academy of Medical Sciences and Sichuan Provincial People's Hospital, School of Medicine, University of Electronic Science and Technology of China, 32 First Ring Road West 2, Chengdu, Sichuan 610072, P.R. China

E-mail: houbin_zhang@yahoo.com

*Contributed equally

Key words: cyclic nucleotide-gated channel subunit alpha 1, autosomal recessive retinitis pigmentosa, whole-exome sequencing variant was absent in other unaffected family members and 600 ethnicity-matched healthy controls. The variant was co-segregated with the disease phenotype in an autosomal recessive manner.

\section{Introduction}

Retinitis pigmentosa (RP) is a group of inherited retinal dystrophies, which is characterized by the progressive loss of function in rod and cone photoreceptors and pigment epithelial cells $(1,2)$. RP affects approximately 1 in 4,000 individuals worldwide (3). Currently, there are $\sim 325,000$ patients in China suffering from RP, and the condition may be inherited in an autosomal-recessive, autosomal-dominant, X-linked or mitochondrial pattern (4-6). To date, $>60$ genes have been identified in non-syndromic RP, including 41 genes associated with an autosomal-recessive form (arRP), according to the RetNet database (https://sph.uth.edu/RetNet). These genes encode for proteins involved in a variety of diverse functional pathways in the neural retina, including photoreceptor development, phototransduction, retinoid cycle, cilia, photoreceptor outer segment development and intracellular protein transport $(3,7)$; dysregulation of these pathways generally results in RP (8). Typical patients with RP display features of night blindness, retinal pigment epithelium atrophy, visual field constriction, pale optic discs and bone spicule pigmentation. Although tremendous progress has been made in the study of RP-associated genes, the identified genes account for merely $60 \%$ of all clinical cases of RP, while the pathogenic variants remain elusive in the remaining cases (9). RP is highly heterogenous, making its accurate diagnosis difficult, and diagnosis only depends on clinical traits for certain cases. In recent years, tremendous advances have been made in molecular biology to identify disease-associated genes for Mendelian disorders. Furthermore, numerous studies demonstrated that exome sequencing is a powerful, efficient method for identifying pathogenic genes of monogenic disorders, including RP (10-12). 
In the present study, whole-exome sequencing (WES) was used to identify the pathogenic variant in a non-consanguineous Chinese family with arRP. A novel homozygous frameshift variant in the cyclic nucleotide-gated channel subunit alpha 1 (CNGA1) gene was identified in the patient with RP.

\section{Materials and methods}

Subjects. A Chinese family was recruited from the First Hospital of Jilin University (Changchun, China), including a total of seven members, with one affected patient and six unaffected individuals (Fig. 1). The control subjects were recruited from Sichuan Provincial People's Hospital (Chengdu, China). All control subjects underwent ophthalmic examination and exhibited no symptoms of RP. The recruitment of the control subjects was previously described (13). The study was implemented in conformity to the tenets of the Declaration of Helsinki and approved by the Institutional Review Boards of Sichuan Provincial People's Hospital (Chengdu, China) and the First Hospital of Jilin University (Changchun, China). Written informed consent was obtained from all participants or parents of children prior to their inclusion in the present study.

Clinical diagnosis. Comprehensive ophthalmic examinations were performed on the proband (age, 47 years), the proband's only daughter (age, 23 years), the proband's three brothers (age, 54,51 and 44 years, respectively), and the proband's only sister (age, 57 years) in the present study. The examinations included visual acuity testing, computerized visual field measurement, tests of dark adaptation and color vision, optical coherence tomography (OCT) and fundus imaging.

DNA isolation. Peripheral blood samples were collected in EDTA tubes from the proband (II:4), the proband's daughter (III:1) and the proband's brothers (II:2, II:3 and II:5). Genomic DNA was extracted using the salt precipitation method, involving lysis, precipitation, washing and resuspension (14). DNA samples were stored at $-20^{\circ} \mathrm{C}$ until use.

WES. The DNA of case no. II:4 (the proband) was analyzed by WES at Novogene Biotechnology Inc., with a mean read depth of target regions of $100 \mathrm{X}$. The sequenced samples were prepared according to the Illumina standard protocol (Illumina, Inc.). First, genomic DNA was randomly fragmented to an average size of $180-280$ bp by a Covaris S220 sonicator (Covaris, Inc.). The remaining overhangs were converted into blunt ends via exonuclease polymerase activities. Subsequently, DNA fragments were end-repaired and phosphorylated, followed by A-tailing and ligation at the 3'ends with paired-end adaptors (Illumina, Inc.). DNA fragments with ligated adapter molecules on both ends were selectively enriched by PCR. After PCR amplification, libraries were hybridized with a liquid phase containing a biotin-labeled probe and magnetic beads with streptomycin were used to capture the exons of genes. Captured libraries were enriched by PCR to add index tags to prepare for sequencing. Products were purified using an AMPure XP system (Beckman Coulter, Inc.) and quantified using the Agilent high-sensitivity DNA assay on the Agilent
I
II

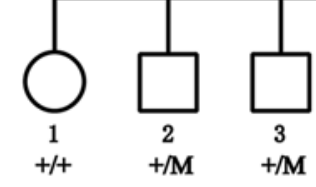

III
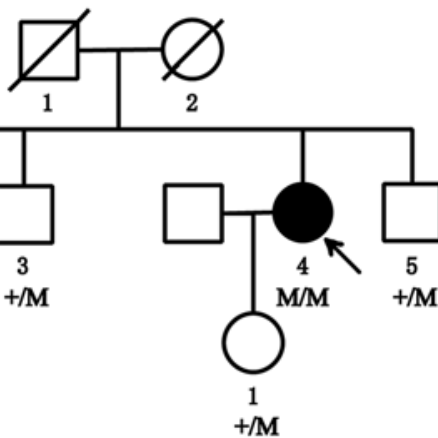

Figure 1. Pedigree of the family with RP in the study. The arrow points to the proband, circles represent females and squares represent males. Empty symbols indicate unaffected status and filled symbols represent affected status. Crossed symbols indicate deceased family members. RP, retinitis pigmentosa; M, mutation [cyclic nucleotide-gated channel subunit alpha 1, c.265delC (p.L89Ffs*3)].
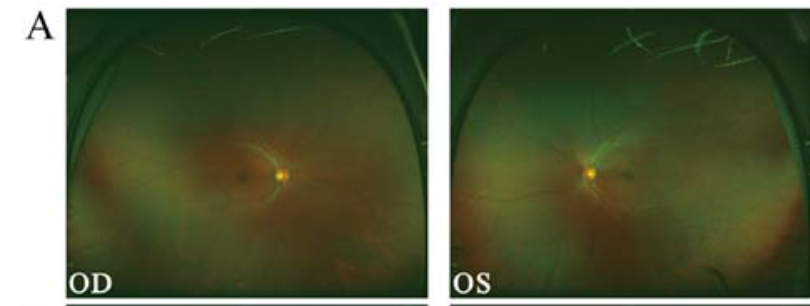

B
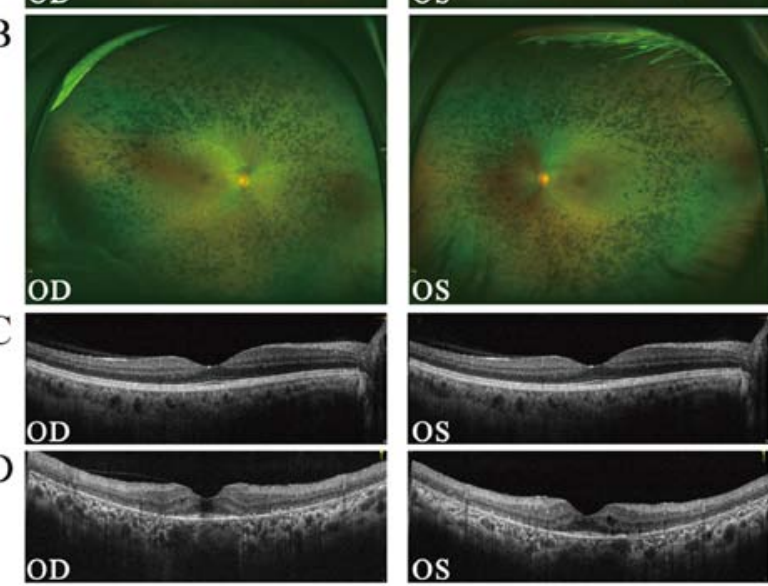

Figure 2. Fundus and OCT images of the proband display typical symptoms of retinitis pigmentosa in the retina. (A) Fundus image of subject no. III:1 from the family. (B) Fundus image of subject no. II:4 (the proband) from the family. (C) OCT of subject no. III:1 from the family. (D) OCT of subject no. II:4 (the proband) from the family. OCT, optical coherence tomography; OS, left eye; OD, right eye.

Bioanalyzer 2100 system (Agilent Technologies, Inc.). Finally, the DNA library was sequenced on Illumina HiSeq4000 for paired-end 150 bp reads.

Variant validation. Sanger sequencing was used to confirm the identified mutation in CNGA1. All exons of CNGA1 were amplified from the genomic DNAs from subjects II:2-5 and III:1 in the recruited family by PCR using standard conditions (15). Amplified products were purified and sequenced with forward and reverse primers (15) by using the BigDye ${ }^{\circledR}$ Terminator v3.1 Cycle Sequencing kit (Applied Biosystems; Thermo Fisher Scientific, Inc.) on a 3730 ABI DNA sequencer (Thermo Fisher Scientific, Inc.). 
A

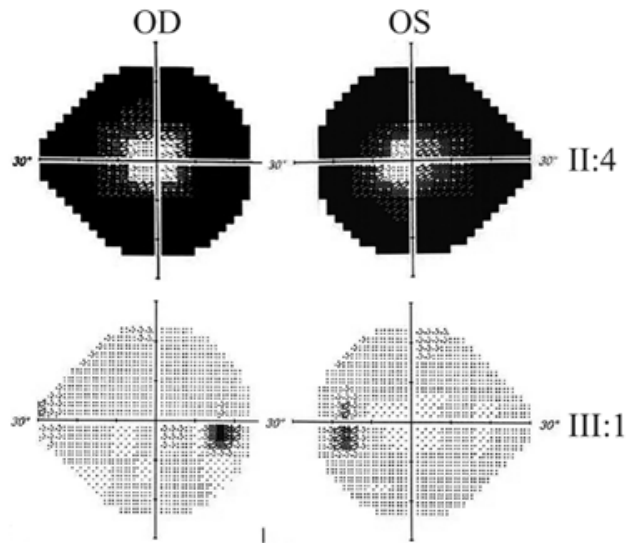

OD

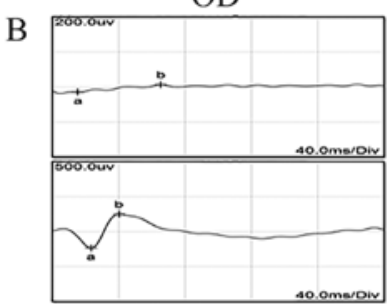

C

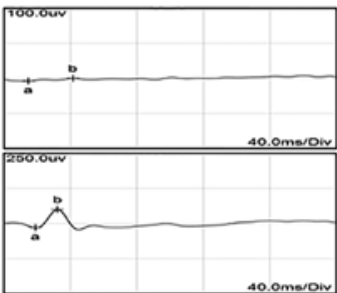

$\mathrm{D}$
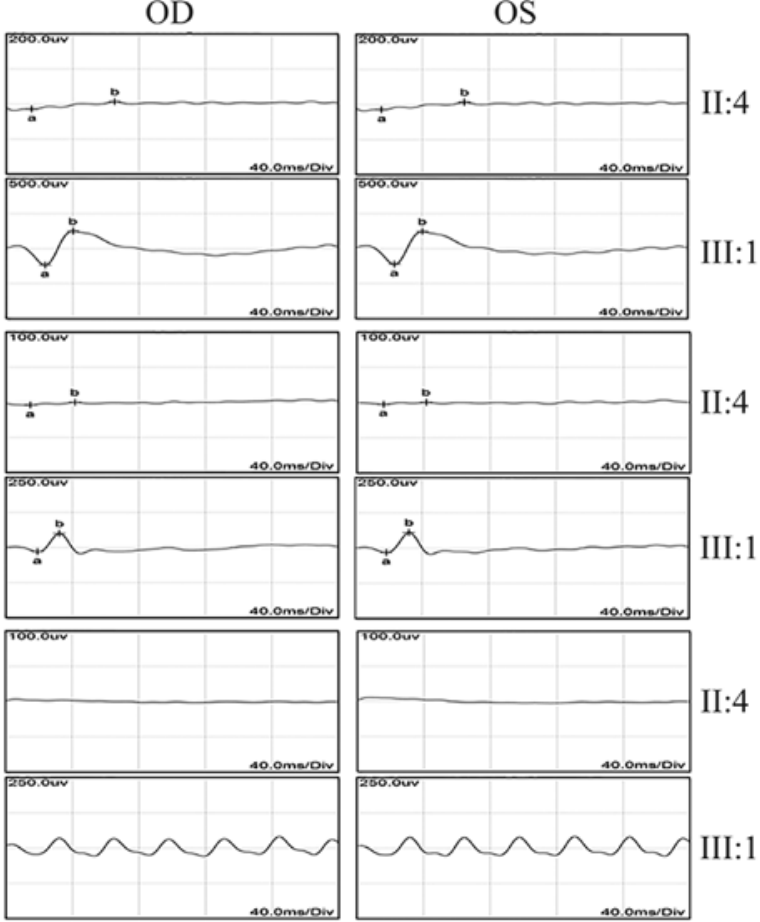

Figure 3. Abnormal retinal function in the proband. (A) Visual field test for subjects II4 and III:1 from the family. The visual fields are severely diminished in subject II:4. (B-D) ERG test for II:4 and III:1 from the family. Subjects II:4 and III:1 from the family were subjected to full-field (B) scotopic ERG, (C) photopic ERG and (D) flicker ERG tests. In the proband, II:4, the ERGs exhibited hardly any response in the scotopic, photopic ERG or flicker ERG tests. Subject III:4 exhibited normal responses in all ERG tests. ERG, electroretinography; OS, left eye; OD, right eye.

\section{Results}

Clinical phenotype. A Chinese family with RP, without any history of consanguineous marriage, was recruited for the present study. The pedigree chart is provided in Fig. 1. Examination of the fundus revealed a normal fundus in subject no. III:1, the proband's daughter (Fig. 2A). By contrast, the proband (II:4) exhibited several classic characteristics of RP, including attenuated blood vessels, pale optic discs and paravascular bone spicule pigmentation (Fig. 2B). OCT revealed thinning of the outer nuclear layer and thinning of the reflex of the retinal pigment epithelium-Bruch's membrane complex (Fig. 2C). The visual field test indicated a significant loss of peripheral vision in the proband, while the vision was normal in the unaffected individuals (II:2, 3, 5 and III:1; Fig. 3A).

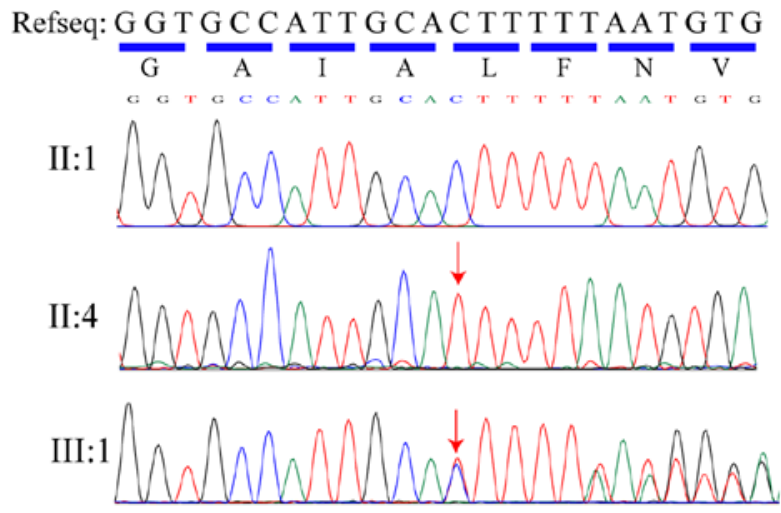

Figure 4. Validation of the variant in CNGA1 by Sanger sequencing. Partial sequence of exon 6 of CNGA1 from an unaffected family member (II:1), the proband (II:4) and a carrier (III:1). The arrows indicate the site of the variant. The Refseq from GenBank and its coding amino acid sequence is displayed at the top. CNGA1, cyclic nucleotide-gated channel subunit alpha 1; Refseq, reference sequence.

Electroretinography (ERG) recordings were barely detectable in the proband under either the scotopic or photopic conditions, indicating an almost complete loss of the function of both rods and cones (Fig. 3B-D). The proband's daughter (III:1) had normal visual responses in the ERG tests. In summary, while all of the healthy unaffected family members had normal vision, the affected individual exhibited the typical clinical symptoms of late-stage RP.

WES and data analysis. To identify the genetic variant causing the RP condition in the family, WES was performed for the proband (subject no. II:4). In total, 24,702 SNPs in coding regions or splicing junctions (2,797 nonsynonymous SNPs, 3,349 synonymous SNPs and 18,556 other types of SNP) and 659 indels were identified. Since neither the proband's parents nor her siblings were affected, the pathogenic variant was more likely to be recessive. Thus, the recessive genetic mode was applied to analyze those SNPs. In order to define the pathogenic variant, the homozygous and compound heterozygous variants were closely followed, particularly frameshift insertions or deletions in the coding region, splicing acceptor and donor site variants, as well as non-synonymous variants that were more likely to be pathogenic. Of note, a homozygous single nucleotide deletion variant in exon 6 of CNGA1 (c.265delC; Chr4:47951883-47951884) was identified in subject no. II:4. This variant causes a frameshift of the open reading frame and loss of function of the protein. CNGA1 is an essential protein involved in the phototransduction cascade in rod photoreceptors. Without CNGA1, the phototransduction cascade would fail in the rods (16). Therefore, this variant was a top candidate subjected to further validation.

Variant validation and co-segregation analysis. The homozygous variant, c.265delC (p.L89Ffs*3) in CNGA1, was confirmed using direct Sanger sequencing in the affected individual (II:4; Fig. 4). It was also determined that, while the proband's daughter (III:1) and one of the proband's brothers (II:2) were heterozygous carriers of c.265delC (p.L89Ffs*3), the other two brothers of the proband (II:3 and II:5) had no variant at this position. The homozygous recessive variant 
matched the genotype/phenotype in this family. Furthermore, this variant was absent from 600 ethnicity-matched normal control samples (median age, 61; age range, 40-78 years) that underwent Sanger sequencing. Therefore, it was concluded that the homozygous CNGA1 c.265delC variant (p.L89Ffs*3) is the variant responsible for the RP condition in the proband.

\section{Discussion}

RP exhibits similar clinical symptoms between cases and ultimately leads to severe blindness, but RP is highly heterogenous from the genetic point of view. The exact clinical symptoms for each patient with RP may vary from case to case. Clinicians may face challenges to diagnose the condition solely based on clinical appearance in certain cases (14). In the past decade, WES has proven successful in efficiently identifying variants from inherited diseases (10-12), markedly improving the accurate diagnosis of RP.

In the present study, a pedigree with RP was screened by using exome sequencing and a homozygous variant, c.265delC (p.L89Ffs*3), was identified in the CNGA1 gene. CNGA1, also known as RP49 or CNCG1, is a member of the subfamily of cyclic nucleotide-gated cation channels. The CNGA1 gene, located at chromosome 4p12, contains 11 exons. CNGA1 encodes the $\alpha$-subunit of the rod cGMP-gated channel (CNG) and is predominantly expressed in rod photoreceptors. CNGA1 consists of six putative transmembrane domains, a pore region and a cGMP-binding domain $(17,18)$. Rod CNG is a multi-subunit protein composed of CNGA1 and CNGB1 at a ratio of 3:1 (19). CNG is a voltage-independent and nonselective ion channel that is activated by the direct binding of cGMP $(20,21)$. CNGs are involved in the final step of the phototransduction cascade $(22,23)$. In addition to its function as a channel protein in the phototransduction cascade, CNGA1 is also required for the formation of the structure of rod photoreceptor outer segments (24).

Variants in the CNGA1 gene have been reported to cause arRP in different ethnic populations $(6,15,25,26)$. The affected individuals with CNGA1 variants display classic features of RP. In the present study, a novel homozygous variant, c.265delC (p.L89Ffs*3) in the CNGA1 gene, was identified in a Chinese family with arRP. PolyPhen2 (genetics.bwh.harvard.edu/pph2/) predicted that the variant may damage the function of the protein. This frameshift variant causes truncation of CNGA1 close to the N-terminus and may lead to complete loss of function. This is consistent with no detectable ERGs in the proband (II:4). Although CNGA1 is exclusively expressed in rods, the affected patient in the present study also exhibited impaired cone functions, as demonstrated by the photopic and flicker ERG. The reason for this may be that rods account for $\sim 95 \%$ of all photoreceptors in the human retina, and in the late stage of RP, substantial rod loss may undermine the physical support of cones provided by rods in the retina. In the absence of this physical support, cones may not function properly and can degenerate progressively. The formation of outer segments may also be affected in this patient. It may be possible to reveal the exact phenotype arising from this variant by an animal model with the CNGA1 frameshift variant (16). To date, there is no treatment available for RP caused by CGNAl mutations. Gene therapy, however, may be a promising treatment in the future.
In conclusion, the present study revealed a novel homozygous variant (p.L89Ffs*3) in the CNGA1 gene in a Chinese family with arRP using WES. The present study demonstrated that WES is a cost-effective method to screen disease-causing variants in arRP. The present results expand the spectrum of known variants associated with RP and may facilitate the molecular diagnosis of RP prior to potential gene therapy for patients carrying this variant in the future.

\section{Acknowledgements}

Not applicable.

\section{Funding}

This research project was supported by the National Precision Medicine Project (grant no. 2016YFC0905200 to HZ), the National Natural Science Foundation of China (grant nos. 81371030,81570882 and 81770935 to HZ), and the Science and Technology of Jilin Province (grant no. 20200201470JC to LW).

\section{Availability of data and materials}

The datasets used and analyzed during the present study are available from the corresponding author on reasonable request.

\section{Authors' contributions}

LW, JH and HBZ conceived the study. TDZ, YQL, PZ and LL performed the experiments. LW recruited the patients. HBZ and BG analyzed the data. TDZ and HBZ wrote the manuscript. All authors read and approved the final manuscript.

\section{Ethics approval and consent to participate}

This study was approved by the Institutional Review Boards of Sichuan Provincial People's Hospital (Chengdu, China) and the First Hospital of Jilin University (Changchun, China). Written informed consent was obtained from all participants or parents of children prior to their inclusion in the present study.

\section{Patient consent for publication}

Written informed consent for publication was obtained from all family members participating in the present study.

\section{Competing interests}

The authors declare that they have no competing interests.

\section{References}

1. Narayan DS, Wood JP, Chidlow G and Casson RJ: A review of the mechanisms of cone degeneration in retinitis pigmentosa. Acta Ophthalmol 94: 748-754, 2016.

2. Zobor D and Zrenner E: Retinitis pigmentosa-A review. Pathogenesis, guidelines for diagnostics and perspectives. Ophthalmologe 109: 501-514; quiz 515, 2012 (In German).

3. Nash BM, Wright DC, Grigg JR, Bennetts B and Jamieson RV: Retinal dystrophies, genomic applications in diagnosis and prospects for therapy. Transl Pediatr 4: 139-163, 2015. 
4. Bunker $\mathrm{CH}$, Berson EL, Bromley WC, Hayes RP and Roderick TH: Prevalence of retinitis pigmentosa in maine. Am J Ophthalmol 97: 357-365, 1984.

5. Grøndahl J: Estimation of prognosis and prevalence of retinitis pigmentosa and usher syndrome in Norway. Clin Genet 31: 255-264, 1987.

6. Paloma E, Martinez-Mir A, Garcia-Sandoval B, Ayuso C Vilageliu L, Gonzàlez-Duarte R and Balcells S: Novel homozygous mutation in the alpha subunit of the rod cGMP gated channel (CNGA1) in two Spanish sibs affected with autosomal recessive retinitis pigmentosa. J Med Genet 39: E66, 2002.

7. Daiger SP, Bowne SJ and Sullivan LS: Perspective on genes and mutations causing retinitis pigmentosa. Arch Ophthalmol 125: 151-158, 2007.

8. Berson EL: Retinitis pigmentosa. The friedenwald lecture. Invest Ophthalmol Vis Sci 34: 1659-1676, 1993.

9. Xu Y, Guan L, Shen T, Zhang J, Xiao X, Jiang H, Li S, Yang J, Jia X, Yin Y, et al: Mutations of 60 known causative genes in 157 families with retinitis pigmentosa based on exome sequencing. Hum Genet 133: 1255-1271, 2014.

10. Ng SB, Buckingham KJ, Lee C, Bigham AW, Tabor HK, Dent KM, Huff CD, Shannon PT, Jabs EW, Nickerson DA, et al: Exome sequencing identifies the cause of a mendelian disorder. Nat Genet 42: 30-35, 2010.

11. Zhao F, Wu J, Xue A, Su Y, Wang X, Lu X, Zhou Z, Qu J and Zhou X: Exome sequencing reveals CCDC111 mutation associated with high myopia. Hum Genet 132: 913-921, 2013.

12. $\mathrm{Hu}$ YS, Song H, Li Y, Xiao ZY and Li T: Whole-exome sequencing identifies novel mutations in genes responsible for retinitis pigmentosa in 2 nonconsanguineous Chinese families. Int J Ophthalmol 12: 915-923, 2019.

13. Liu X, Wu Y, Miao Z, Zhang H, Gong B, Zhu X, Huang L, Shi Y, Hao F, Ma S, et al: A novel deletion downstream of the PAX6 gene identified in a Chinese family with congenital aniridia. Ophthalmic Genet 39: 428-436, 2018.

14. Wang M, Gan D, Huang X and Xu G: Novel compound heterozygous mutations in CNGAlin a Chinese family affected with autosomal recessive retinitis pigmentosa by targeted sequencing. BMC Ophthalmol 16: 101, 2016.

15. Zhang Q, Zulfiqar F, Riazuddin SA, Xiao X, Ahmad Z, Riazuddin S and Hejtmancik JF: Autosomal recessive retinitis pigmentosa in a Pakistani family mapped to CNGA1 with identification of a novel mutation. Mol Vis 10: 884-889, 2004

16. Hüttl S, Michalakis S, Seeliger M, Luo DG, Acar N, Geiger H, Hudl K, Mader R, Haverkamp S, Moser M, et al: Impaired channel targeting and retinal degeneration in mice lacking the cyclic nucleotide-gated channel subunit CNGB1. J Neurosci 25 $130-138,2005$
17. Dhallan RS, Macke JP, Eddy RL, Shows TB, Reed RR, Yau KW and Nathans J: Human rod photoreceptor cGMP-gated channel: Amino acid sequence, gene structure, and functional expression. J Neurosci 12: 3248-3256, 1992.

18. Pittler SJ, Lee AK, Altherr MR, Howard TA, Seldin MF, Hurwitz RL, Wasmuth JJ and Baehr W: Primary structure and chromosomal localization of human and mouse rod photoreceptor cGMP-gated cation channel. J Biol Chem 267: 6257-6262, 1992.

19. Shuart NG, Haitin Y, Camp SS, Black KD and Zagotta WN: Molecular mechanism for 3:1 subunit stoichiometry of rod cyclic nucleotide-gated ion channels. Nat Commun 2: 457, 2011.

20. Craven KB and Zagotta WN: CNG and HCN channels: Two peas, one pod. Annu Rev Physiol 68: 375-401, 2006.

21. Fesenko EE, Kolesnikov SS and Lyubarsky AL: Induction by cyclic GMP of cationic conductance in plasma membrane of retinal rod outer segment. Nature 313: 310-313, 1985.

22. Yau KW: Phototransduction mechanism in retinal rods and cones. The friedenwald lecture. Invest Ophthalmol Vis Sci 35: 9-32, 1994

23. Dryja TP, Finn JT, Peng YW, McGee TL, Berson EL and Yau KW: Mutations in the gene encoding the alpha subunit of the rod cGMP-gated channel in autosomal recessive retinitis pigmentosa. Proc Natl Acad Sci USA 92: 10177-10181, 1995.

24. Tosi J, Davis RJ, Wang NK, Naumann M, Lin CS and Tsang SH: shRNA knockdown of guanylate cyclase 2e or cyclic nucleotide gated channel alpha 1 increases photoreceptor survival in a cGMP phosphodiesterase mouse model of retinitis pigmentosa. J Cell Mol Med 15: 1778-1787, 2011.

25. Iwanami M, Oshikawa M, Nishida T, Nakadomari S and Kato S: High prevalence of mutations in the EYS gene in Japanese patients with autosomal recessive retinitis pigmentosa. Invest Ophthalmol Vis Sci 53: 1033-1040, 2012.

26. Gonzalez-del Pozo M, Borrego S, Barragan I, Pieras JI, Santoyo J, Matamala N, Naranjo B, Dopazo J and Antiñolo G: Mutation screening of multiple genes in Spanish patients with autosomal recessive retinitis pigmentosa by targeted resequencing. PLoS One 6: e27894, 2011

This work is licensed under a Creative Commons Attribution-NonCommercial-NoDerivatives 4.0 International (CC BY-NC-ND 4.0) License. 